\title{
Extraction of the bare form factors for the semi-leptonic $B_{s}$ decays
}

\author{
F. Bahr, D. Banerjee, M. Koren*, H. Simma, R. Sommer \\ NIC, DESY, Platanenallee 6, D-15738 Zeuthen, Germany \\ E-mail: felixtbahr@googlemail.com, debasish.banerjee@desy.de, \\ mateusz.koren@desy.de, hubert.simma@desy.de, rainer.sommer@desy.de
}

\begin{abstract}
The computation of the form factors for the $\mathrm{B}_{\mathrm{s}} \rightarrow \mathrm{K} \ell v$ decay is presented. The $\mathrm{b}$ quark is treated by means of Heavy Quark Effective Theory, currently in the static approximation. In these proceedings we discuss the extraction of the bare matrix elements from lattice data through a combined fit to two- and three-point correlation functions, as well as by considering suitable ratios. The different methods agree concerning the extracted form factors and approximately $2 \%$ accuracy is reached. The non-perturbative renormalization and matching to QCD is described in accompanying proceedings [1].
\end{abstract}

34th annual International Symposium on Lattice Field Theory

24-30 July 2016

University of Southampton, UK

\footnotetext{
* Speaker.
} 


\section{Introduction: definitions, ensembles, measurements}

The QCD matrix elements for the semi-leptonic $\mathrm{B}_{\mathrm{s}} \rightarrow \mathrm{K} \ell v$ decay in the rest frame of the $\mathrm{B}_{\mathrm{s}}$ meson are

$$
\begin{aligned}
& \left(2 m_{\mathrm{B}_{\mathrm{s}}}\right)^{-1 / 2}\left\langle\mathrm{~K}\left(\vec{p}_{\mathrm{K}}\right)\left|V^{0}(0)\right| \mathrm{B}_{\mathrm{s}}(0)\right\rangle=h_{\|}\left(E_{\mathrm{K}}\right), \\
& \left(2 m_{\mathrm{B}_{\mathrm{s}}}\right)^{-1 / 2}\left\langle\mathrm{~K}\left(\vec{p}_{\mathrm{K}}\right)\left|V^{k}(0)\right| \mathrm{B}_{\mathrm{s}}(0)\right\rangle=p_{\mathrm{K}}^{k} h_{\perp}\left(E_{\mathrm{K}}\right),
\end{aligned}
$$

where $V^{\mu}(x)=\bar{\psi}_{\mathrm{u}}(x) \gamma^{\mu} \psi_{\mathrm{b}}(x)$ and $\vec{p}_{\mathrm{K}}$ is the Kaon momentum.

The $b$ quark is treated in the framework of Heavy Quark Effective Theory (HQET) where a full non-perturbative renormalization program exists [2]. Here we focus on the extraction of the bare HQET matrix elements from the lattice data, while the procedure to obtain the QCD form factors from these bare matrix elements is described in [1].

For the heavy quark we use HYP1 and HYP2 discretizations [3]. We first restrict ourselves to the static approximation. The two- and three-point functions of interest are

$$
\begin{aligned}
\mathscr{C}^{\mathrm{K}}\left(t_{\mathrm{K}} ; \vec{p}_{\mathrm{K}}\right) & =\sum_{t_{i}} \sum_{\vec{x}_{f}, \vec{x}_{i}} e^{-i \vec{p}_{\mathrm{K}} \cdot\left(\vec{x}_{f}-\vec{x}_{i}\right)}\left\langle P_{\mathrm{su}}\left(\vec{x}_{f}, t_{i}+t_{\mathrm{K}}\right) P_{\mathrm{us}}\left(\vec{x}_{i}, t_{i}\right)\right\rangle, \\
\mathscr{C}^{\mathrm{B}_{\mathrm{s}}}\left(t_{\mathrm{B}_{\mathrm{s}}} ; \overrightarrow{0}\right) & =\sum_{t_{i}} \sum_{\vec{x}_{f}, \vec{x}_{i}}\left\langle P_{\mathrm{sb}}\left(\vec{x}_{f}, t_{i}+t_{\mathrm{B}_{\mathrm{s}}}\right) P_{\mathrm{bs}}\left(\vec{x}_{i}, t_{i}\right)\right\rangle, \\
\mathscr{C}_{\mu}^{\mathrm{B}_{\mathrm{s}} \rightarrow \mathrm{K}}\left(t_{\mathrm{K}}, t_{\mathrm{B}_{\mathrm{s}}} ; \vec{p}_{\mathrm{K}}\right) & =\sum_{t_{i}} \sum_{\vec{x}_{f}, \vec{x}_{v}, \vec{x}_{i}} e^{-i \vec{p}_{\mathrm{K}} \cdot\left(\vec{x}_{f}-\vec{x}_{v}\right)}\left\langle P_{\mathrm{su}}\left(\vec{x}_{f}, t_{i}+t_{\mathrm{B}_{\mathrm{s}}}+t_{\mathrm{K}}\right) V_{\mu}\left(\vec{x}_{v}, t_{i}+t_{\mathrm{B}_{\mathrm{s}}}\right) P_{\mathrm{bs}}\left(\vec{x}_{i}, t_{i}\right)\right\rangle,
\end{aligned}
$$

with $P_{\mathrm{q}_{1} \mathrm{q}_{2}}(\vec{x}, t)=\bar{\psi}_{\mathrm{q}_{1}}(\vec{x}, t) \gamma_{5} \psi_{\mathrm{q}_{2}}(\vec{x}, t)$. For the light quarks we use Wuppertal smearing $[4,5]$. The Kaon correlator is calculated with only one level of smearing, while for the $\mathrm{B}_{\mathrm{s}}$ meson we apply three levels of smearing (in both the two- and three-point functions). We can decompose the Euclidean correlation functions as

$$
\begin{aligned}
\mathscr{C}^{\mathrm{K}}\left(t_{\mathrm{K}}\right) & =\sum_{m}\left(\kappa^{(m)}\right)^{2} e^{-E_{\mathrm{K}}^{(m)} t_{\mathrm{K}}}, \\
\mathscr{C}_{i j}^{\mathrm{B}_{\mathrm{s}}}\left(t_{\mathrm{B}_{\mathrm{s}}}\right) & =\sum_{n} \beta_{i}^{(n)} \beta_{j}^{(n)} e^{-E_{\mathrm{B}_{\mathrm{s}}}^{(n)} t_{\mathrm{B}_{\mathrm{s}}}}, \\
\mathscr{C}_{\mu, i}^{\mathrm{B}_{\mathrm{s}} \rightarrow \mathrm{K}}\left(t_{\mathrm{K}}, t_{\mathrm{B}_{\mathrm{s}}}\right) & =\sum_{n, m} \kappa^{(m)} \varphi_{\mu}^{(m, n)} \beta_{i}^{(n)} e^{-E_{\mathrm{K}}^{(m)} t_{\mathrm{K}}} e^{-E_{\mathrm{B}_{\mathrm{s}}}^{(n)} t_{\mathrm{B}}},
\end{aligned}
$$

where the indices $m, n$ label the Kaon and $\mathrm{B}_{\mathrm{s}}$ meson energy levels respectively, while the indices $i, j$ label the smearing levels used for the $\mathrm{B}_{\mathrm{s}}$ meson. In our current setup $\vec{p}_{K}$ has a non-zero component only in the $x$-direction, therefore we only extract the form factors for $\mu=0,1$. Thus, the desired static bare matrix elements are given by

$$
h_{\|}^{\text {stat,bare }}=\varphi_{0}^{(0,0)} \sqrt{2 E_{\mathrm{K}}^{(0)}}, \quad p_{\mathrm{K}}^{1} h_{\perp}^{\text {stat,bare }}=\varphi_{1}^{(0,0)} \sqrt{2 E_{\mathrm{K}}^{(0)}} .
$$

We use three $N_{f}=2$ CLS ensembles [6]: A5, F6, and N6, which have similar pion mass $\left(m_{\pi}=310-340 \mathrm{MeV}\right)$ but different lattice spacings ( $a \approx 0.075,0.065$ and $0.048 \mathrm{fm}$ respectively), allowing us to take the continuum limit. For further details on the ensembles, see Table 2 of Ref. [7].

We choose $\left|\vec{p}_{\mathrm{K}}\right|=0.535 \mathrm{GeV}$ which corresponds to $2 \pi / L$ on the N6 lattice. We keep the same value of $\vec{p}_{\mathrm{K}}$ on the other lattices by introducing flavour-twisted boundary conditions [8] for the strange quark (cf. Ref. [7]). 
Computing all-to-all propagators with a random source on every timeslice ("full time dilution") allows us to access all time separations in the two-point and three-point functions. For more details on the measurements and analysis we refer to the upcoming Ref. [9].

\section{Bare form factor extraction by means of a combined fit}

Our goal is to extract the form factors by fitting to the three-point correlation function, Eq. (1.8). We first determine the parameters of the two-point correlation functions, Eqs. (1.6), (1.7), and use these as fixed input to estimate the form factors $\varphi_{\mu}$ from a linear fit to Eq. (1.8). We then use all these parameters as initial values to a "global" combined fit to Eqs. (1.6)-(1.8). We find that in this way one obtains superior stability of the fit results with respect to small changes of the initial values and fit ranges.

Our interest is mostly limited to the ground-state form factors $\varphi_{\mu}^{(0,0)}$, however we find that for the safe extraction, free of contamination by the excited states, we need to include more terms in the sums of Eqs. (1.6)-(1.8). In these proceedings we keep only the Kaon ground state but include two excited states for the $\mathrm{B}_{\mathrm{s}}$ meson.

Clearly, a good choice for the initial values and a careful choice of fit ranges is required to obtain stable combined fits. Let us briefly describe how this is done in the following subsections.

\subsection{Two-point function fits}

Eq. (1.6) for the two-point light-light correlator is taken in the limit of infinite $T$. In practice, our lattices have finite $T$ and one has to take into account the wrap-around state, giving

$$
\mathscr{C}^{\mathrm{K}}(t) \cong\left(\kappa^{(0)}\right)^{2}\left(e^{-E_{\mathrm{K}}^{(0)} t}+e^{-E_{\mathrm{K}}^{(0)}(T-t)}\right),
$$

when $t$ and $T-t$ are large enough that we can neglect the contribution of the excited states.

We select the time $t_{\min }^{\mathrm{K} 2}$ at which we start the fit by choosing the smallest value of $t$ at which the (fitted) excited-state contribution is smaller than $1 / 4$ of the statistical uncertainty at that value of $t$. There is no severe signal-to-noise problem in the Kaon sector, so we always use $t_{\max }^{\mathrm{K} 2}=T / 2$.

For the two-point heavy-light $\left(\mathrm{B}_{\mathrm{S}}\right)$ correlator, we have three different smearings. Including the off-diagonal terms yields six independent correlators in the symmetric $\mathscr{C}_{i j}^{\mathrm{B}_{\mathrm{s}}}$ matrix. We first obtain the energies using GEVP, with $t_{0}=\lceil t / 2\rceil$ (cf. Refs. [10,5]). Then we determine the amplitudes in Eq. (1.7) by first doing a linear fit to the diagonal elements of $\mathscr{C}^{\mathrm{B}}$ to find the squares of the amplitudes and then using these values as input to the non-linear fit for $\beta_{i}^{(n)}$ to all elements of $\mathscr{C}_{i j}^{\mathrm{B}_{\mathrm{s}}}$, including the off-diagonal ones.

\subsection{Safeguarding from finite- $T$ contributions in $\mathscr{C}^{\mathbf{B}_{\mathrm{s}} \rightarrow \mathbf{K}}$}

Due to the finite time extent of the lattice, we have to take into account the "wrappers" in the three-point functions - at large enough times $\mathscr{C}_{\mu, i}^{\mathrm{B}_{\mathrm{s}} \rightarrow \mathrm{K}}$ can be written as a sum of the ground-state contributions to the two diagrams shown in Fig. 1:

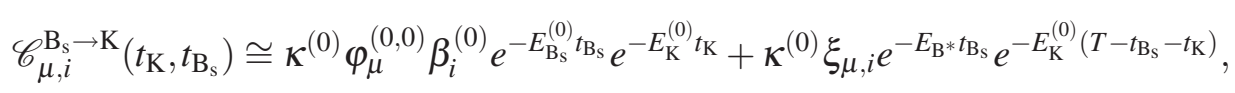



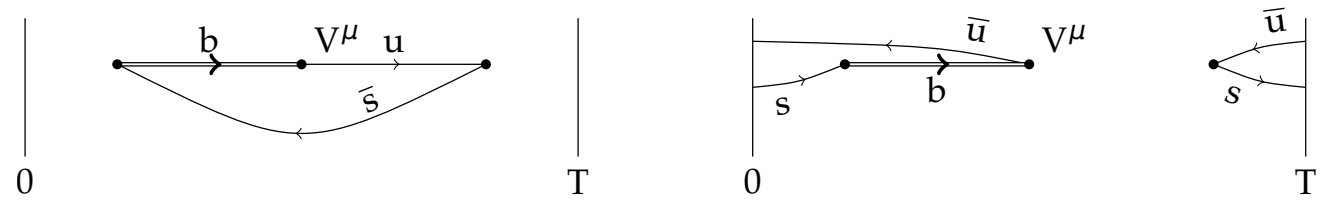

Figure 1: Physical (left) and wrapper (right) contributions to $\mathscr{C}^{\mathrm{B}_{\mathrm{s}} \rightarrow \mathrm{K}}$.

where $\xi_{\mu, i}=\left\langle 0\left|V_{\mu}\right| \mathrm{B}^{*}\right\rangle\left\langle\mathrm{B}^{*}\left|P_{\mathrm{hl}}\right| \mathrm{K}\right\rangle$ is the unknown matrix element of the wrapper state and $E_{\mathrm{B}^{*}}$ is the energy of the lightest heavy-light state contributing to the wrapper diagram. In the static order we have $E_{\mathrm{B}^{*}}=E_{\mathrm{B}_{\mathrm{s}}}^{(0)}$, but at NLO it will be different.

To include the wrappers in the fit we need at least six extra parameters $\xi_{\mu, i}$. Instead we choose to exclude these states by restricting the fit region so that their contribution is negligible. To do that, for every given value of $t_{\mathrm{B}_{\mathrm{s}}}, \mu$, and $i$ we fit the three point function to the form

$$
\mathscr{C}_{\mu, i}^{\mathrm{B}_{\mathrm{s}} \rightarrow \mathrm{K}}\left(t_{\mathrm{K}}, t_{\mathrm{B}_{\mathrm{s}}}\right) \cong B_{\mu, i} e^{-E_{\mu, i}^{\mathrm{K}} t_{\mathrm{K}}}+C_{\mu, i} e^{+E_{\mu, i}^{\mathrm{K}} t_{\mathrm{K}}}
$$

with $B_{\mu, i}$ and $C_{\mu, i}$ being linear fit parameters (which one can express in terms of the amplitudes and matrix elements of Eq. (2.2)) and $E_{\mu, i}^{\mathrm{K}}$ being a non-linear fit parameter. Then we find $t_{\mathrm{max}, \mathrm{wr}}^{\mathrm{K} 3}$ as the last $t_{\mathrm{K}}$ for which the fitted wrapper contribution to the function is smaller than $1 / 3$ of its statistical uncertainty at that value of $t_{\mathrm{K}}$. Final $t_{\max }^{\mathrm{K} 3}\left(t_{\mathrm{B}_{\mathrm{s}}}\right)$ can be chosen as the minimum of $t_{\text {max,wr }}^{\mathrm{K} 3}\left(t_{\mathrm{B}_{\mathrm{s}}}\right)$ and $t_{\text {max,noise }}^{\mathrm{K} 3}\left(t_{\mathrm{B}_{\mathrm{s}}}\right)$, where the latter excludes the points with a relative statistical error of $\mathscr{C}^{\mathrm{B}_{\mathrm{s}} \rightarrow \mathrm{K}}\left(t_{\mathrm{K}}, t_{\mathrm{B}_{\mathrm{s}}}\right)$ larger than 0.1 .

The resulting $t_{\max }^{\mathrm{K} 3}\left(t_{\mathrm{B}_{\mathrm{s}}}\right)$ curves for the N6 ensemble are plotted in Fig. 2. One clearly observes that the wrapper contamination is more pronounced for $\mu=1$.

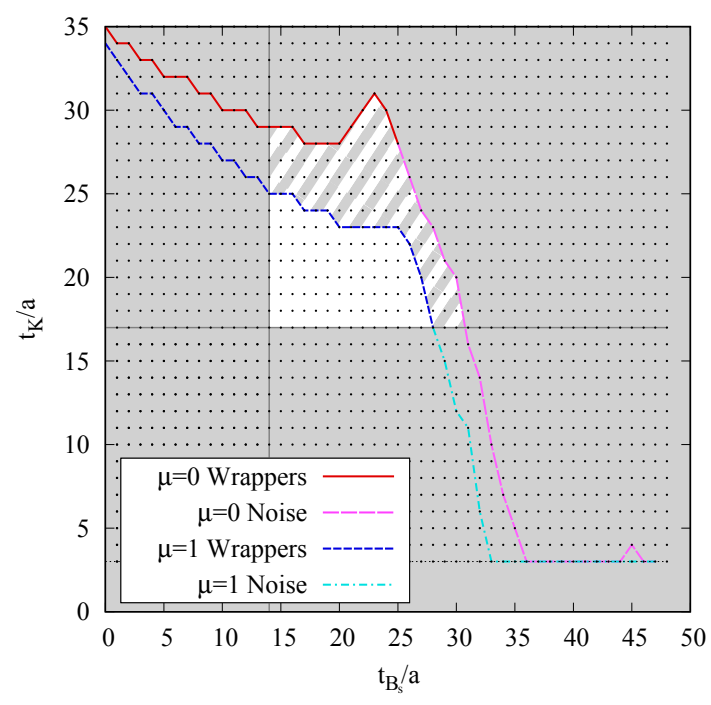

Figure 2: The lines of $t_{\max }^{\mathrm{K} 3}$ for $\mu=0$ and $\mu=1$. The regions disallowed for the fits (with $t_{\mathrm{min}}^{\mathrm{K} 3}, t_{\mathrm{min}}^{\mathrm{B} 3}$ from [7]) are in gray. The region allowed for $\mu=0$ but disallowed for $\mu=1$ is in white-gray stripes.

\subsection{Combined fit, stability}

Having determined the parameters in Eqs. (1.6), (1.7) and the estimates for the form factors $\varphi_{\mu}$ from linear fits to Eq. (1.8), we use them as initial values for the combined non-linear fit, i.e. we simultaneously fit the three equations for all values of $\mu$ and all light-quark smearings for the $\mathrm{B}_{\mathrm{S}}$ meson.

The temporal fit ranges are determined by suitable criteria described above, except for three minimum times: $t_{\min }^{\mathrm{B} 2}, t_{\min }^{\mathrm{B} 3}$ and $t_{\min }^{\mathrm{K} 3}$, which in our setup are independent of $\mu$ and the smearing level. They are chosen such that the contributions from the excited states are negligible.

We check that the change of the fit results with respect to variations of the fit ranges is negligible within the statistical errors, plotting the results on the "stability plots". An example stability plot for the ground-state matrix elements is presented in Fig. 3. 

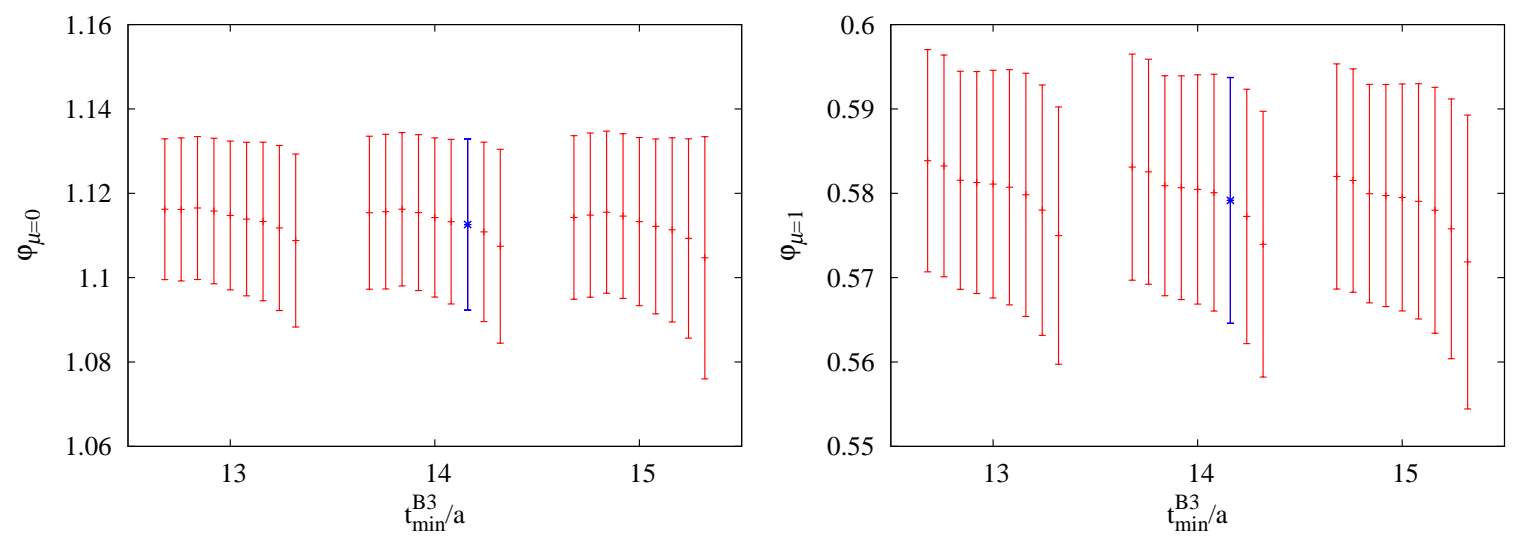

Figure 3: Stability of the fit parameters $\varphi_{0}^{(0,0)}$ (left) and $\varphi_{1}^{(0,0)}$ (right) on ensemble N6 (HYP2 discretization) with respect to variations of $t_{\min }^{\mathrm{B} 3} / a$ (different groups) and of $t_{\min }^{\mathrm{K} 3} / a=11 \ldots 19$ (within the groups). In the plot we fix $t_{\min }^{\mathrm{B} 2}=t_{\min }^{\mathrm{B} 3}-5 a$. In each panel, the value used to determine the bare form factor is marked with a filled square.

\section{Bare form factor extraction by the ratio method}

To cross-check the results obtained by the combined fit, we use the ratio method. One can define many different ratios that converge to the desired form factor in the limit of large $t_{\mathrm{K}}, t_{\mathrm{B}_{\mathrm{S}}}$. Here, we consider three definitions $\left(\tau=t_{\mathrm{K}}+t_{\mathrm{B}_{\mathrm{s}}}\right)$ :

$$
\begin{aligned}
& \mathscr{R}_{\mu, i}^{\mathrm{I}}\left(t_{\mathrm{K}}, t_{\mathrm{B}_{\mathrm{s}}}\right)=\frac{\mathscr{C}_{\mu, i}^{\mathrm{B}_{\mathrm{s}} \rightarrow \mathrm{K}}\left(t_{\mathrm{K}}, t_{\mathrm{B}_{\mathrm{s}}}\right)}{\left[\mathscr{C}^{\mathrm{K}}(\tau) \mathscr{C}_{i i}^{\mathrm{B}_{\mathrm{s}}}(\tau)\right]^{1 / 2}} \mathrm{e}^{\left(\tilde{E}_{\mathrm{B}_{\mathrm{s}}}-\tilde{E}_{\mathrm{K}}\right) \frac{t_{\mathrm{B}_{\mathrm{s}}}-t_{\mathrm{K}}}{2}}, \\
& \mathscr{R}_{\mu, i}^{\mathrm{II}}\left(t_{\mathrm{K}}, t_{\mathrm{B}_{\mathrm{s}}}\right)=\frac{\mathscr{C}_{\mu, i}^{\mathrm{B}_{\mathrm{s}} \rightarrow \mathrm{K}}\left(t_{\mathrm{K}}, t_{\mathrm{B}_{\mathrm{s}}}\right)}{\left[\mathscr{C}^{\mathrm{K}}\left(t_{\mathrm{K}}\right) \mathscr{C}_{i i}^{\mathrm{B}_{\mathrm{s}}}\left(t_{\mathrm{B}_{\mathrm{s}}}\right)\right]^{1 / 2}} \mathrm{e}^{\tilde{E}_{\mathrm{B}_{\mathrm{s}}} \frac{t_{\mathrm{B}}}{2}+\tilde{E}_{\mathrm{K}} \frac{t_{\mathrm{K}}}{2}}, \\
& \mathscr{R}_{\mu, i}^{\mathrm{III}}\left(t_{\mathrm{K}}, t_{\mathrm{B}_{\mathrm{s}}}\right)=\frac{\mathscr{C}_{\mu, i}^{\mathrm{B}_{\mathrm{s}} \rightarrow \mathrm{K}}\left(t_{\mathrm{K}}, t_{\mathrm{B}_{\mathrm{s}}}\right)}{\mathscr{N}^{\mathrm{K}} \mathscr{C}^{\mathrm{K}}\left(t_{\mathrm{K}}\right) \mathscr{N}_{i}^{\mathrm{B}_{\mathrm{s}}} \mathscr{C}_{i i}^{\mathrm{B}_{\mathrm{s}}}\left(t_{\mathrm{B}_{\mathrm{s}}}\right)},
\end{aligned}
$$

and restrict ourselves to the highest $\mathrm{B}_{\mathrm{s}}$ smearing $^{1}$, and the case $t_{\mathrm{K}}=t_{\mathrm{B}_{\mathrm{s}}}=t$.

Ratio $\mathscr{R}^{\mathrm{I}}(t, t)$ has a particularly simple form and needs no extra parameters - it however comes at the price of working with $\mathscr{C}^{\mathrm{B}_{\mathrm{s}}}(2 t)$ in the denominator, which results in more noisy behaviour at large $t$.

For $\mathscr{R}$ II we find that one gets good statistical precision of the results when using $\tilde{E}_{\mathrm{K}}=E_{\mathrm{K}}^{(0)}$, $\tilde{E}_{\mathrm{B}_{\mathrm{s}}}=E_{\mathrm{B}_{\mathrm{s}}}^{(0)}$ obtained from the two-point function fits as described in the previous section. Also for $\mathscr{R}^{\mathrm{III}}$ we use the fitted amplitudes $\mathscr{N}^{\mathrm{K}}=1 / \kappa^{(0)}$ and $\mathscr{N}_{i}^{\mathrm{B}_{\mathrm{s}}}=1 / \beta_{i}^{(0)}$.

The results for the finest lattice spacing are presented in Fig. 4. We see that $\mathscr{R}^{\mathrm{II}}$ and $\mathscr{R}^{\mathrm{III}}$ are nicely consistent with the combined fit results in the vicinity of $0.8 \mathrm{fm} \lesssim t \lesssim 1 \mathrm{fm}$. $\mathscr{R}_{\mu=0}^{\mathrm{I}}$ has superior behaviour for small $t$ but $\mathscr{R}_{\mu=1}^{\mathrm{I}}$ does not and it becomes very noisy before reaching the plateau due to the noise in $\mathscr{C}^{\mathrm{B}_{\mathrm{s}}}(2 t)$.

\footnotetext{
${ }^{1}$ We also analyzed GEVP ratios, following Refs. [10, 11], but found no significant improvement.
} 

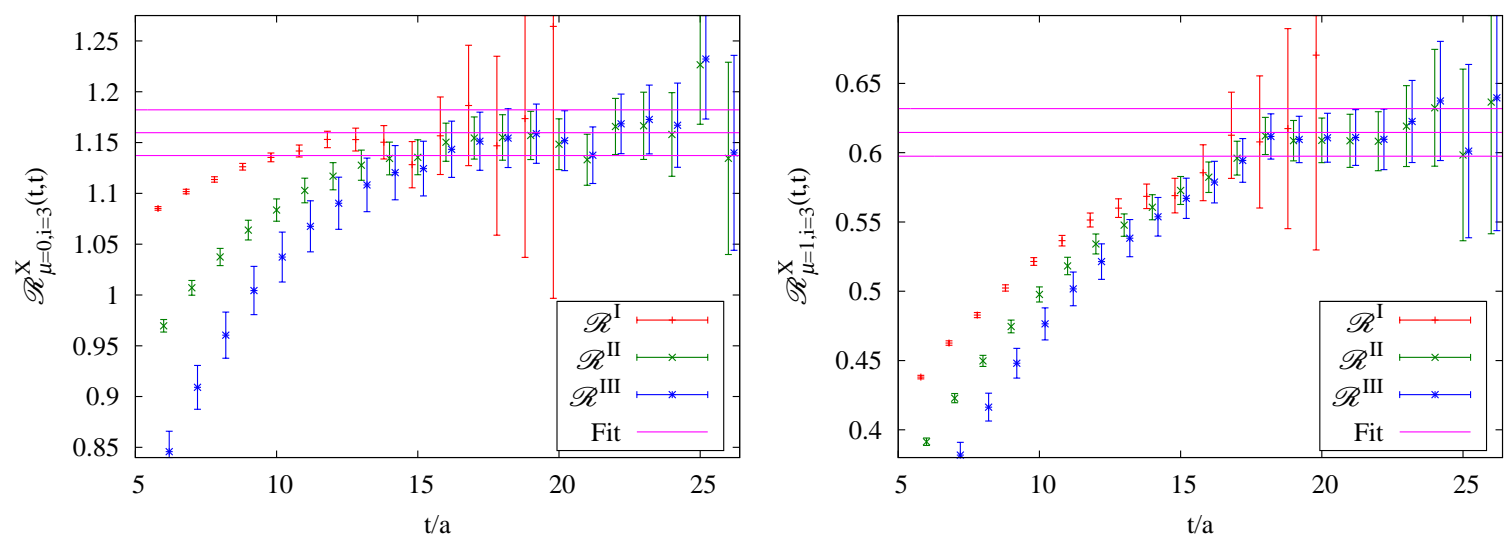

Figure 4: Different ratios $\mathscr{R}_{\mu, i=3}^{X}(t, t)$ for the N6 ensemble (HYP1 discretization): $\mu=0$ (left) and $\mu=1$ (right), together with the combined fit results. Results are slightly displaced on the horizontal axis for better visibility.
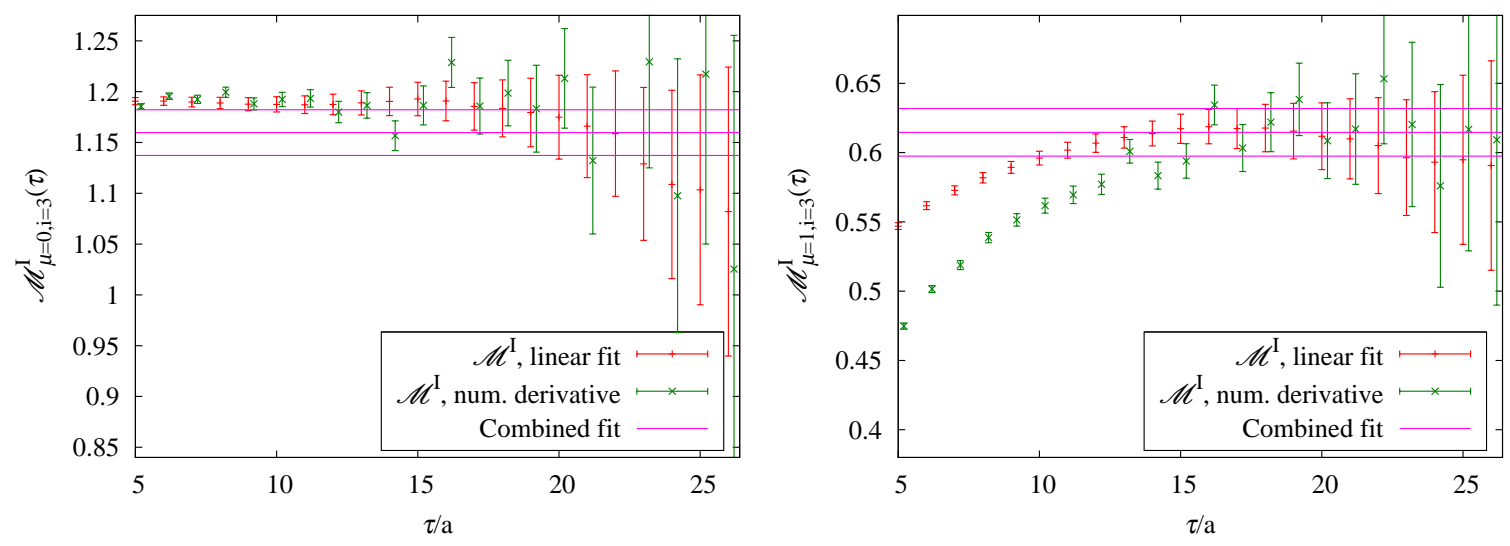

Figure 5: Summed ratios $\mathscr{M}_{\mu, i=3}^{I}(t, t)$ for the N6 ensemble (HYP1 discretization): $\mu=0$ (left) and $\mu=1$ (right), obtained using two methods described in text. The linear fit is done from $\tau$ to $\tau_{\max }=32 a$.

Improved convergence for $\mathscr{R}^{\mathrm{I}}$ can be obtained by summing the ratio [12]:

$$
\mathscr{M}_{\mu, i}^{I}(\tau)=\partial_{\tau} a \sum_{t_{\mathrm{B}}} \mathscr{R}_{\mu, i}^{\mathrm{I}}\left(\tau-t_{\mathrm{B}_{\mathrm{s}}}, t_{\mathrm{B}_{\mathrm{s}}}\right) .
$$

The asymptotic excited-state contaminations are then $\mathscr{O}\left(\tau \Delta \mathrm{e}^{-\tau \Delta}\right)$, where $\Delta=\min \left(E_{\mathrm{K}}^{(1)}-E_{\mathrm{K}}^{(0)}\right.$, $\left.E_{\mathrm{B}_{\mathrm{s}}}^{(1)}-E_{\mathrm{B}_{\mathrm{s}}}^{(0)}\right)$, as opposed to $\mathscr{O}\left(\mathrm{e}^{-\tau \Delta / 2}\right)$ in ordinary ratios [11].

In practice, the derivative can be calculated numerically or one can obtain $\mathscr{M}_{\mu}^{I}$ from a linear fit to the sum (setting $\tau_{\max }$ small enough to avoid the influence of the wrappers). Example results showing the two methods are presented in Fig. 5. We observe that in fact the convergence is improved.

\section{Conclusions and outlook}

We extract the bare matrix elements of the semi-leptonic $\mathrm{B}_{\mathrm{s}}$ decay in the static order of HQET. In this framework, renormalization and matching to QCD can be performed non-perturbatively, such that the continuum limit can be taken, as described in Refs. [1, 7]. 
Our numerical setup allows us to access all time separations in the two-point and three-point correlation functions of interest, therefore giving us a very good handle on the excited-state and finite- $T$ contributions.

To extract the matrix elements from the lattice data, we use two different extraction methods: the combined fit and the ratio method. They give consistent results which makes us confident in the robustness of the analysis. The precision of our results for the bare matrix elements is approx. $2 \%$. The final resulting precision of the static continuum-extrapolated RGI form factors is better than $5 \%$ [7].

We estimate the systematic error from neglecting the subleading terms in $1 / m_{\mathrm{b}}$ to be of order $15 \%$. Therefore, the next step is to include the $1 / m_{\mathrm{b}}$ terms, which will reduce this systematic error to $1-2 \%$. The required set of HQET parameters is being calculated in the parallel effort by the ALPHA collaboration [13].

We also plan to include ensembles with a smaller pion mass, although we expect the effects of the quark mass to be below our uncertainties in the case of the $\mathrm{B}_{\mathrm{s}} \rightarrow \mathrm{K} \ell v$ decay.

\section{References}

[1] D. Banerjee, "Form factors in the $B_{s} \rightarrow K \ell v$ decays using HQET and the lattice", PoS(LATTICE2016) 292.

[2] J. Heitger and R. Sommer, “Nonperturbative heavy quark effective theory”, JHEP 0402 (2004) 022, [arXiv:hep-lat/0310035].

[3] M. Della Morte, A. Shindler, and R. Sommer, "On lattice actions for static quarks", JHEP 0508 (2005) 051, [arXiv:hep-lat/0506008].

[4] S. Güsken et al., "Nonsinglet axial vector couplings of the baryon octet in lattice QCD", Phys. Lett. B227 (1989) 266.

[5] F. Bernardoni et al., "The b-quark mass from non-perturbative $N_{f}=2$ Heavy Quark Effective Theory at $O\left(1 / m_{h}\right)$ ", Phys. Lett. B730 (2014) 171, [arXiv:1311.5498].

[6] P. Fritzsch et al., "The strange quark mass and Lambda parameter of two flavor QCD", Nucl.Phys. B865 (2012) 397, [arXiv:1205.5380].

[7] F. Bahr et al., "Continuum limit of the leading-order HQET form factor in $B_{s} \rightarrow K \ell v$ decays", Phys. Lett. B757 (2016) 473, [arXiv:1601.04277].

[8] P. F. Bedaque, "Aharonov-Bohm effect and nucleon nucleon phase shifts on the lattice", Phys. Lett. B593 (2004) 82, [arXiv:nucl-th/0402051].

[9] F. Bahr, D. Banerjee, M. Koren, H. Simma, and R. Sommer, in preparation.

[10] B. Blossier et al., "On the generalized eigenvalue method for energies and matrix elements in lattice field theory", JHEP 0904 (2009) 094, [arXiv:0902.1265].

[11] J. Bulava, M. Donnellan, and R. Sommer, "On the computation of hadron-to-hadron transition matrix elements in lattice QCD”, JHEP 01 (2012) 140, [arXiv:1108.3774].

[12] L. Maiani et al., "Scalar densities and baryon mass differences in lattice QCD with Wilson fermions", Nucl. Phys. B293 (1987) 420.

[13] M. Della Morte et al., "Non-perturbative matching of HQET heavy-light axial and vector currents in $N_{f}=2$ lattice $Q C D ”$, PoS(LATTICE2016) 199. 